\title{
Intratrial response speed in fixed-ratio behavior: A comparison of positive and negative reinforcement
}

\author{
NAPOLEON C. POZULP II and PETER C. SENKOWSKI \\ Northern Ilinois University, DeKalb, Illinois 60115
}

\begin{abstract}
Terminal decrements in response speeds within trials have been reported in a number of discrete-trial runway and leverpress situations using appetitive reinforcers. One recent attempt to explain this phenomenon suggests that such decrements are the result of the occurrence of premature consummatory behaviors which interfere with the ongoing response sequence. According to this hypothesis, terminal decrements in response speed should not occur in a fixed-ratio discrete trial escape situation where consummatory responses by the subject are not required to gain reinforcement. In support of the hypothesis, terminal decrements in response speed were obtained in an appetitively motivated discrete-trial leverpress situation but not in one in which aversive motivation was employed.
\end{abstract}

A homogeneous behavior sequence (HBS) may be defined as a series of highly similar stimulus-response units terminating in reinforcement. Examples of situations closely approximating HBSs include running in a straight-alley runway and discrete-trial leverpressing on fixed-ratio (FR) reinforcement schedules.

Hull (1934) was one of the first to show a theoretical interest in performance within HBSs. Using his goal-gradient hypothesis, Hull predicted that response speed in a runway would be an inverse function of the distance from the goal. Hull's results indicated that, while running speed did increase over initial sections of the runway, rats showed a decrement in speed as they approached the goal area. Similar decrements in response speed have been observed in both runway (e.g., DiLollo \& Walker, 1964) and discrete-trial leverpress situations (e.g., Platt, 1972; Platt \& Senkowski, 1970; Weiss, 1961). Hull assumed that the terminal decrement in response speed was due to an anticipatory form of the stopping necessary to consume the reinforcer. It has also been suggested that subjects slow down as they approach the end of the runway to avoid hitting the goalbox wall. The latter interpretation, while applicable to the runway situation, does not seem appropriate for explaining the terminal decrements in response speed found in discrete-trial leverpressing situations where no such barrier to continued responding exists.

An alternative explanation of intratrial terminal speed decrements was proposed by Platt and Senkowski (1970). Their hypothesis suggests that accumulated response-correlated stimuli (RCS) serve two functions in an HBS. The RCS are assumed to serve as secondary reinforcers, strengthening the responses which precede them so as to yield a negatively accelerated increasing intratrial speed gradient. The RCS are also assumed to

Requests for reprints should be sent to Napoleon Pozulp, Department of Psychology, Northern mlinois University, DeKalb, Illinois 60115. This paper is sponsored by W. R. McAllister who takes full editorial responsibility for it. act as discriminative stimuli for responses appropriate to consuming the reinforcer $\left(R_{T} s\right)$. With each successive response in the HBS, the accumulated RCS more closely approximate those present at the time of reinforcement and thus have a greater likelihood of eliciting $R_{T} s$ prematurely. It is the premature occurrence of $R_{T} s$, and the resultant interference with the ongoing response sequence, that is assumed to account for the terminal decrements in intratrial speed.

If the Platt and Senkowski (1970) hypothesis is correct, it should be possible to produce an intratrial speed gradient having little or no terminal decrement by using a reinforcer which has no apparent $R_{T} S$ associated with it. An escape paradigm using termination of electric shock as the reinforcer seems to meet this requirement, since completion of the response sequence results in immediate reinforcement. The present experiment was designed to test the adequacy of the Platt and Senkowski hypothesis through the comparison of the intratrial speed gradients generated by discrete-trial, FR reinforcement schedules terminating in either food delivery or offset of electric shock. The Platt and Senkowski hypothesis would predict a nonmonotonic asymptotic response-speed gradient when food serves as the reinforcer, and a monotonic gradient when the reinforcer is termination of shock.

\section{METHOD}

\section{Subjects}

Sixteen experimentally naive, 140-day-old male hooded rats from a colony maintained by the Department of Psychology served as subjects. Rats were randomly and equally assigned to the escape and appetitive groups, which were differentiated on the basis of the type of reinforcer to be used during training. For rats in the escape group, food and water were continuously available except during experimental sessions. Subjects assigned to the appetitive group were reduced to $85 \%$ of ad-lib body weight by restricting their daily food intake and were maintained at this level throughout the experiment. Subjects were treated in 
accordance with ethical standards of the APA.

\section{Apparatus}

A $25.5 \times 23.7 \times 29.0 \mathrm{~cm}$ stainless steel test chamber, contained in a sound-attenuating enclosure, housed subjects during experiment sessions. The height of the chamber was reduced to $14.0 \mathrm{~cm}$ through the use of a Plexiglas insert to prevent posturings by rats in the escape group which could result in the attenuation of shock.

A Foringer retractable lever, requiring approximately $60-\mathrm{g}$ static force to operate, was centered on the subject panel, $4.5 \mathrm{~cm}$ above the grid floor. A Lehigh Valley pellet dispenser was used to bait a recessed food tray which was mounted $2 \mathrm{~cm}$ directly above the lever opening. Two $28-\mathrm{V}$ dc pilot lamps were mounted on the subject panel $7.0 \mathrm{~cm}$ above the lever opening and $1.6 \mathrm{~cm}$ apart. For rats in the escape group, scrambled shock was supplied to the grid floor, lever, and chamber wails, by a Grason-Stadler Model 700 shock generator.

Electromechanical circuitry was used to program experimental contingencies. Response latencies and interresponse times (IRTs) were recorded on impulse counters to $.1 \mathrm{sec}$. All circuitry and recording equipment was located in a room adjacent to the experimental chamber to reduce noise.

\section{Procedure}

Following initial leverpress training, subjects received $10 \mathrm{FR}$ trials per day using a discrete-trial procedure. A trial began with insertion of the lever, brightening of the pilot lamps, and, for rats in the escape group, onset of a nominal $1.6-\mathrm{mA}$ shock. Completion of the response requirement resulted in lever retraction, offset of the pilot lamps, and termination of shock for escape-group subjects or delivery of a single .045-g food pellet for subjects in the appetitive group. A $60-\mathrm{sec}$ intertrial interval then ensued prior to the start of the next trial.

Beginning with a FR 1 , the size of the ratio was gradually increased until a FR 8 response requirement was reached. Data collection then began and continued until a total of 32 test sessions had been administered.

\section{RESULTS}

Latencies of the first response and IRTs for each of the remaining seven responses of the FR were averaged over the 10 trials of each session for each subject. Reciprocals of these values were calculated to obtain speed measures, and these values were averaged over blocks of 40 trials. Average interresponse speeds as a function of ordinal response position within the ratio for both groups on the last block of trials is presented in Figure 1.

Since the speed of the first response in an HBS is influenced by the subject's orientation with regard to the lever at the time the trial begins, it is not completely comparable with the other responses in the sequence. Therefore, separate analyses were performed on first-response speeds and speeds of the remaining responses of the FR. It was found that the speed of the first response on the last block of training trials was reliably faster for subjects in the escape group than for those in the appetitive group, $\mathrm{F}(1,14)=18.88, \mathrm{p}<.001$. This difference was present on the first block of trials and persisted throughout the experiment. Speeds of the remaining responses of the FR for the last block of training trials were subjected to an analysis of variance in which the between-subjects factor was groups (escape vs. appetitive) and the within-subjects factor was ordinal response position (Positions 2-8). The effect of ordinal response position was dependent upon the reinforcer used as indexed by a reliable groups by ordinal response position interaction, $F(6,84)=2.72, p<.025$. This interaction was further investigated through the use of treatment by subject analyses of variance performed on the data from each group. The results of these analyses indicated that while ordinal response position had no effect on response speeds in the escape group, $F<1$, this variable had a highly reliable effect on speeds of the appetitive group, $F(6,42)=5.30, p<.001$. Inspection of Figure 1 suggests that the interresponse speed gradient for the appetitive group is nonmonotonic, having the form of an inverted $U$. Supporting this impression were the results of a subsequent test on trends using data from the appetitive group, which revealed a highly reliable quadratic component, $F(1,42)=22.08$, $\mathrm{p}<.001$, in the variance attributable to ordinal response position.

Although the present experiment was concerned primarily with potential differences in asymptotic response speed gradients between groups, preasymptotic data were analyzed and can be easily summarized. Interresponse speeds were found to increase over training in both groups. This increase was accompanined by a change in gradient shape for the appetitive group, but not for the escape group. The shape of the gradient for the escape group shown in Figure 1 is representative of that gradient's form throughtout training. In the appetitive group, the highest interresponse speeds early in training occurred at about the second ordinal response position with speeds declining throughout the remainder of the ratio. With increased training in this group, the

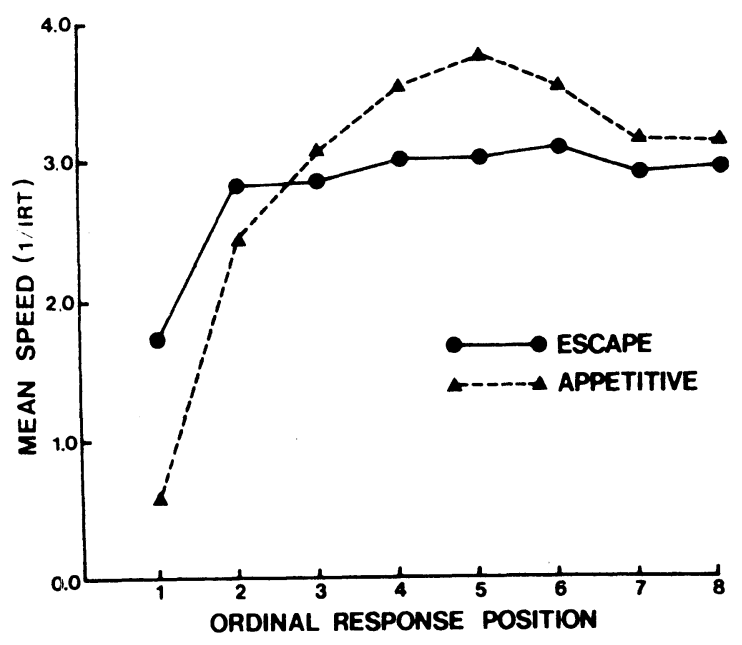

Figure 1. Mean asymptotic interresponse speeds as a function of ordinal response position within the FR for groups receiving either shock termination or a single food pellet as reinforcement. 
peak speed moved toward the middle of the ratio and settled at about the fifth ordinal position as shown in Figure 1. This shift in maximum intratrial response speed is predicted by the Platt and Senkowski (1970) hypothesis.

\section{DISCUSSION}

The Platt and Senkowski (1970) account of behavior within HBSs assumes that terminal decrements in intratrial response speed are the result of the premature elicitation of $\mathbf{R}_{T}$ s by RCS, which interfere with the ongoing behavior sequence. It was hypothesized that use of a reinforcer which requires no $R_{T} s$ would result in an interresponse speed gradient having no terminal decrement. The results of the present experiment support this prediction, in that terminal decrements in intratrial speeds were obtained when food was the reinforcer but not when the reinforcer was termination of shock. Evidence concerning the importance of $\mathrm{R}_{\mathrm{T}} \mathrm{s}$ in determining performance within HBSs would be further supported if it can be shown that variables seen as affecting the vigor and/or magnitude of $R_{T}$ could also be shown to affect the shape of interresponse speed gradients. The effects of delay and magnitude of reward on the shape of intratrial speed gradients are currently being investigated in our laboratory.

\section{REFERENCES}

DiLollo, V., \& Walker, E. L. Speed and basal resistance level (BRL) in a segmented straight alley. Psychological Record 1964, 14, 499-505.

Hull, C. L. The rat's speed-of-locomotion gradient in an approach to food. Journal of Comparative Psychology, 1934, $17,393-422$.

Platt, J. R. Amount of training, deprivation, and variability of chain length as determinants of response velocity gradients in homogeneous chains. Journal of Experimental Psychology, 1972, 92, 191-197.

Platt, J. R., \& Senkowski, P. C. Response-correlated stimulus functioning in homogeneous behavior chains. In J. H Reynierse (Ed.), Current issues in animal learning. Lincoln: University of Nebraska Press, 1970.

Weiss, R. F. Response speed, magnitude, and resistance to extinction as joint function of work and length of behavior chain. Journal of Experimental Psychology, 1961, 61, 245-256.

(Received for publication December 9, 1974.) 